Case Report

\title{
Fatal cerebral gas embolism after hemodialysis
}

\begin{abstract}
We report the case of a 74 year-old-woman who after hemodialysis session developed hemodynamic instability, coma and decerebrate posture, secondary to severe cerebral vessels gas embolism.
\end{abstract}

Keywords: Gas embolism, Stroke, Hemodialysis
Volume 2 Issue 5 - 2015

\section{Carlo Piero Lastarria Perez}

Neurologist, National Hospital Carlos Alberto Seguín Escobedo, Peru

Correspondence: Dr. Carlo Piero Lastarria Perez, Neurologist, National Hospital Carlos Alberto Seguín Escobedo, ESSALUD, Arequipa, Peru, Email carlolastarria@hotmail.com

\section{Introduction}

Gas embolism can result from procedures performed in almost all clinical specialties. Gas embolism is a rare complication of the use of hemodialysis. Its incidence is about 1 in 2000 episodes. ${ }^{1}$ We report a patient who after hemodialysis experienced neurological damage from gas embolism. It was evidenced by brain computed tomography (CT) scan showing multiple cerebral gas embolisms.

\section{Case presentation}

A 74 year-old-woman with a history of chronic nephropathy in hemodialysis and high blood pressure, she came to the hospital for hemodialysis session. When the hemodialysis session was near to end, she collapsed.

She was transferred to the shock trauma unit. Her vital signs were blood pressure 200/100, sinus tachycardia of 110/min, oxygen saturation of $95 \%$, respiratory frequency of $16 / \mathrm{min}$. At neurological examination, the patient was in coma with mydriatic, isochoric and active pupils.

In response to painful stimuli she developed a decerebrate posture consisting of rigid extension of the arms and legs, plantar flexion of the ankles, and pronation of the forearms. Babinski reflexes were present. Nuchal rigidity was absent. Cardiovascular and pulmonary exam were normal. The patient was intubated and went to the CT room for urgent brain tomography. CT revealed air embolism principally on venous vessels on right hemisphere (Figure $1 \& 2$ ).

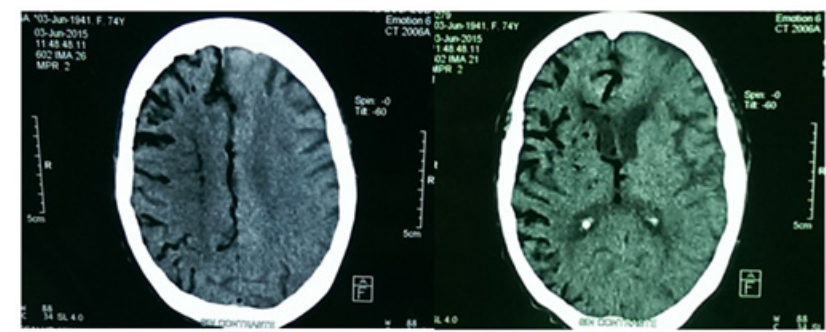

Figure I NCCT head demonstrating massive air embolism in the right hemispherical vessels.

Minutes later, patient becomes hypotensive with BP 70/50 $\mathrm{mmHg}$. In laboratory results, WBC count, haemoglobin, platelets, glucose and INR were in normal levels. Sodium and potassium were unremarkable. Creatinine was high in $4,3 \mathrm{mg} / \mathrm{dl}$ and urea in $70 \mathrm{mg} /$ dl. Gas blood analysis showed pO2 of $286 \mathrm{mmHg}$ with a FiO2 1.0 and mild respiratory alkalosis. One hour later the condition of patient got worst and she passed away.

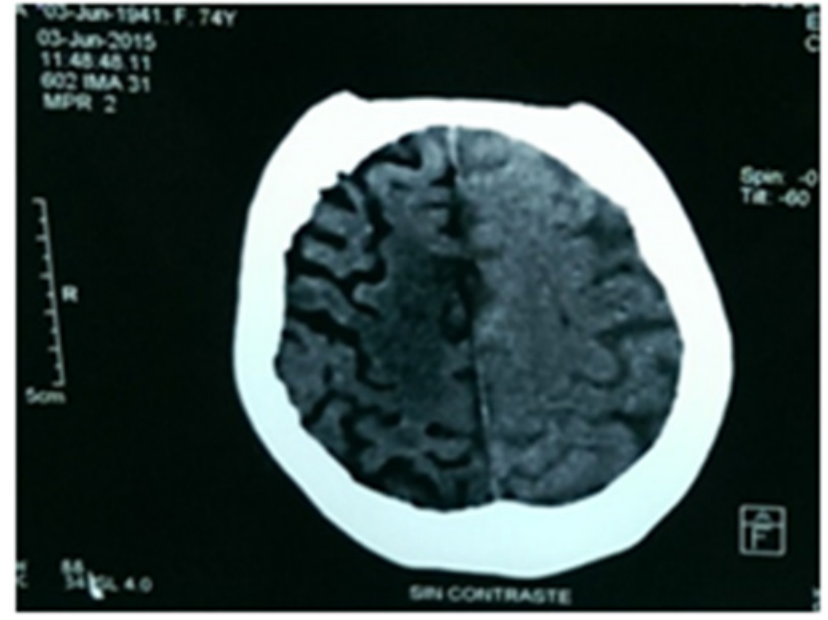

Figure 2 On right frontal parasagittal area we can observe a hypodense area due to ischemic damage.

\section{Discussion}

Gas embolism on arterial or venous circulation is a very dangerous pathology with high rate of morbidity and mortality. Gas embolism is related to many specialities. It can be caused by central venous catheterization, mechanical ventilation with high pressure, chest trauma, thoracentesis, hemodialysis, and several other invasive vascular procedures ${ }^{2,3}$ (Figure 3 ).

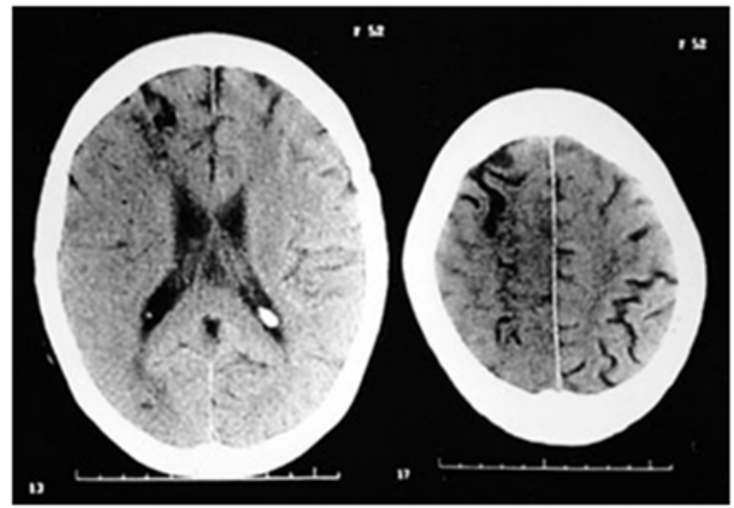

Figure 3 Air embolism in the watershed area between anterior and middle right cerebral arteries as a complication of endoscopy procedure (a) A communication of source of air and the vessel (b) a pressure gradient that pushes air into the circulation. 
Air embolism in the watershed area, between anterior and middle right cerebral arteries has a complication of endoscopy procedure. ${ }^{4}$

There are two crucial factors for gas embolism to occur:

a. A communication of source of air and the vessel.

b. A pressure gradient that pushes air into the circulation. ${ }^{5}$

Volumes between 200 to $300 \mathrm{ml}$ or $3-5 \mathrm{~mL} / \mathrm{kg}$ of air in humans for significant injury to occur have been described. ${ }^{6}$ But the injection of only 2 or $3 \mathrm{~mL}$ of air into the cerebral circulation can be fatal and it also depends of proximity of vessel to the right heart, because if it is closer, it could be necessary a smaller volume of air to be fatal. ${ }^{7}$

If there is an anomalous communication between right and left circulation as it occurs in atrial or ventricular septal defect, patent foramen ovale, or arterial venous malformations or fistula for hemodialysis, arterial embolism can occur as a complication of venous embolism. ${ }^{8}$

The pulmonary capillaries are able to filter out the air bubbles, but if the volume exceeds $30 \mathrm{ml}$ or air bubbles have more than $22 \mathrm{um}$ of diameter, the mechanism of depuration is insufficient, this could happen in barotrauma. ${ }^{9,10}$

Clinical presentations mimic cardiac, pulmonary and neurologic dysfunctions. Because of this a high index of suspicion is necessary to establish the diagnosis and institute the adequate treatment. ${ }^{11}$ Cardiovascular and respiratory failure and vascular insufficiency of central nervous system could be observed in severe cases of gas emboli. It is also important the body position of patient at the time of the event. If the patient is in a sitting position, gas will travel retrograde via the internal jugular vein to the cerebral circulation, causing neurologic symptoms. ${ }^{12}$ This can include decreased consciousness, seizures, focal motor deficits, collapse, and coma. Other system symptoms include arrhythmia, hypotension, circulatory shock, tachypnea. ${ }^{11}$

When the gas enters into the systemic circulation it occludes the microcirculation and causes ischemic damage to multiple organs, including brain, heart and lung. Embolism also releases inflammatory mediators and oxygen free radicals. ${ }^{11}$

Air bubbles greater than $1.3 \mathrm{~mm}$ are could be identified in the brain CT. ${ }^{10,13}$ To detect gas in the ventricles and the presence of a patent foramen ovale echocardiography may be useful. Routine laboratory tests are performed to evaluate the associated end-organ injury resulting from air embolism.

Arterial blood gas exam often can show hypoxemia, hypercapnia and metabolic acidosis. This pathology could mimic a pulmonary embolism, with hypoxia, decreased PCO2 levels, and respiratory alkalosis. ${ }^{11}$

Management of gas embolism once is suspected includes:

a. Endotracheal intubation is recommended for severe respiratory distress or in a comatose patient in order to maintain adequate oxygenation and ventilation. High flow (100\%) oxygen will help to reduce the bubble's nitrogen content and therefore size. ${ }^{12}$

b. Lateral decubitus or Trendelenburg position helps to prevent air from travelling through the right side of the heart into the pulmonary arteries. ${ }^{12,14}$

c. If patient has neurological manifestations and cardiovascular instability hyperbaric oxygen therapy facility could improve prognosis. The benefit mechanism include compression of existing bubbles, because a high diffusion gradient to speed resolution of existing bubbles, improved oxygenation of ischemic tissues, and lowered intracranial pressure. This therapy may be used in the first 6 hours of event. ${ }^{15}$

d. Fluids and vasopressors can be used as supportive therapy. Mechanical ventilation may be necessary. ${ }^{16}$

e. In some animal studies, perfluorocarbons (FP-43) have been shown to facilitate reabsorption of bubbles, decreasing neurologic and cardiovascular complications. ${ }^{6}$

\section{Conclusion}

In conclusion stroke due to gas embolism could be a fatal pathology and the suspicion is necessary to establish the diagnosis and institute the adequate treatment, especially in a hospital with a hemodialysis service.

As we reported in the case, our patient had the two crucial requisites to present gas embolism: A communication of source of air and the vessel and a pressure gradient that pushes air into circulation. Both represented by the connection of hemodialysis machine and the patient's fistula.

Moreover, as it was said before, the patient had a fistula for hemodialysis and this is a risk factor for arterial embolism as a complication of venous embolism as it is mentioned in the literature.

In this patient the principal manifestation of the disease was the collapse followed by the decerebrate posture. This signs in addition of the history of recent hemodialysis were the key for the clinical suspicion. Finally it was confirmed with the brain CT. Unfortunately we did not have a hyperbaric oxygen therapy facility that could improve prognosis of the patient.

\section{Acknowledgments}

None.

\section{Conflicts of interest}

None.

\section{References}

1. Ward MK, Shadforth M, Hill AV, et al. Air embolism during haemodialysis. Br Med J. 1971;3(5766):74-78.

2. Van Hulst RA, Klein J, Lachmann B. Gas embolism: pathophysiology and treatment. Clin Physiol Funct Imaging. 2003;23(5):237-246.

3. Moon RE. Air or Gas Embolis. Hyperbaric Oxygen Committee Report. 2003;pp.5-10.

4. Mellado TP, Constanzo P F, Miquel P JF, et al. Ischemic brain infarction after an air embolism. Case report. Rev Med Chil. 2005;133(4):453-456. [Article in Spanish].

5. Muth CM, Shank ES. Gas embolism. N Engl J Med. 2000;342(7):476482 .

6. Mirski MA, Lele AV, Fitzsimmons L, et al. Diagnosis and treatment of vascular air embolism. Anesthesiology. 2007;106(1):164-177.

7. Ho AM. Is emergency thoracotomy always the most appropriate immediate intervention for systemic air embolism after lung trauma? Chest. 1999;116(1):234-237.

8. Platz E. Tangential Gunshot Wound to the Chest Causing Venous Air Embolism: A Case Report and Review. J Emerg Med. 2008;41(2):e25e29. 
9. Murphy BP, Harford FJ, Cramer FS. Cerebral air embolism resulting from invasive medical procedures.Treatment with hyperbaric oxygen. Ann Surg. 1985;201(2):242-245.

10. Weiss KL, Macura KJ, Ahmed A. Cerebral air embolism: acute imaging. J Stroke Cerebrovasc Dis. 1998;7(3):222-226.

11. http://emedicine.medscape.com/article/761367-clinical\#b1

12. Sheasgreen J, Terry T, Mackey JR. Large--volume air embolism as a complication of augmented computed tomography: case report. Can AssocRadiol J. 2002;53(4):199-201.

13. Ghatge SB, Bhatgadde VL, Nagar AM, et al. Paradoxical cerebral arterial gas embolism: Computed tomography findings. Australas Radiol. 2007;51Suppl:B210-B213.
14. Karaosmanoglu D, Oktar SO, Araç M, et al. Case report: Portal and systemic venous gas in a patient after lumbar puncture. $\mathrm{Br} J$ Radiol. 2005;78(932):767-769.

15. Ohashi S, Endoh H, Honda T, et al. Cerebral air embolism complicating percutaneous thin-needle biopsy of the lung: complete neurological recovery after hyperbaric oxygen therapy. J Anesth. 2001;15(4):233236 .

16. Archer DP, Pash MP, MacRae ME. Successful management of venous air embolism with inotropic support. Can J Anaesth. 2001;48(2):204 208 